\title{
Processing and Interpretation Times of CT Angiogram and CT Perfusion in Stroke
}

\author{
Ashok Srinivasan, Mayank Goyal, Cheemun Lum, Thanh Nguyen, William Miller
}

\begin{abstract}
Objective: To determine the mean time for acquiring computed tomogram perfusion (CTP) and CT angiogram (CTA) images in acute stroke. To determine and compare processing and interpretation times amongst three groups of radiologists with varying degree of expertise: two radiology residents (Group I), two neuroradiology fellows (Group II) and four consultant neuroradiologists (Group III). Methods: The mean time of acquisition of CTA and CTP studies was calculated among ten patients presenting with acute stroke. All readers had to process the CTA and CTP images, interpret them (for presence or absence of thrombus and penumbra) and save them on the GE Advantage Windows workstation. The mean time for processing and interpreting these studies was calculated. Results: The mean time for acquisition of CTA and CTP studies in the ten patients was $14.6 \pm 5.9$ minutes. The time taken for CTA processing and interpretation in Groups I, II and III was $2.3 \pm 1.3 \mathrm{~min}, 1.6 \pm 0.4 \mathrm{~min}$ and $1.5 \pm 0.7 \mathrm{~min}$ respectively. The time required for CTP processing and interpretation by the same groups was $5.2 \pm 1.7 \mathrm{~min}, 4.5 \pm 1.5 \mathrm{~min}$ and $4.1 \pm 1.1 \mathrm{~min}$ respectively. There was a statistically significant difference of means between Groups I and III in the CTA and CTP processing and interpretation times ( $\mathrm{p}=0.02$, $\mathrm{p}=0.01$ respectively) but no statistical difference between Groups I and II ( $\mathrm{p}=0.15, \mathrm{p}=0.22$ respectively) or Groups II and III ( $\mathrm{p}=0.31$, $\mathrm{p}=0.30$ respectively). Conclusion: The CTA and CTP studies can be performed, processed and interpreted quickly in acute stroke.
\end{abstract}

RÉSUMÉ: Temps de traitement et d'interprétation de l'angiographie CT et de la perfusion CT dans l'accident vasculaire cérébral. Objectif: Déterminer le temps moyen d'acquisition des images au moyen du CT avec perfusion (CTP) et de l'angiographie CT (CTA) dans l'accident vasculaire cérébral aigu (AVCA). Déterminer et comparer le temps de traitement et d'interprétation par trois groupes de radiologistes ayant un degré d'expertise variée: 2 résidents en radiologie (groupe I), 2 étudiants gradués en neuroradiologie (groupe II) et 4 neuroradiologistes consultants (groupe III). Méthodes: Le temps moyen d'acquisition des images par CTA et CTP a été calculé chez dix patients porteurs d'un AVC aigu. Tous les radiologistes devaient traiter les images CTA et CTP, les interpréter pour déterminer la présence ou l'absence de thrombus ou de pénombre et les enregistrer sur la station de travail Advantage Windows Workstation de GE. Le temps moyen de traitement et d'interprétation de ces études a été calculé. Résultats: Le temps moyen d'acquisition des études CTA et CTP chez les dix patients était de 14,6 \pm 5,9 minutes. Le temps de traitement et d'interprétation des images obtenues par CTA dans les groupes I, II et III était de 2,3 $\pm 1,3$ minutes, 1,6 $\pm 0,4$ minutes et 1,5 $\pm 0,7$ minutes respectivement. Le temps de traitement et d'interprétation des images obtenues par CTP était de 5,2 $\pm 1,7$ minutes, 4,5 $\pm 1,5$ minutes et 4,1 $\pm 1,1$ minutes respectivement. Il y avait une différence significative au point de vue statistique entre les moyennes des groupes I et III pour le temps de traitement et d'interprétation $(\mathrm{p}=0,02$ et $\mathrm{p}=0,01$ respectivement), mais pas de différence entre les groupes I et II ( $p=0,15$ et $p=0,22$ respectivement), ou les groupes II et III ( $p=0,31$ et $p=0,30$ respectivement). Conclusion: Les études par CTA et CTP peuvent être effectuées, traitées et interprétées rapidement dans l'AVCA.

Can. J. Neurol. Sci. 2005; 32: 483-486

Timely administration of intravenous and/or intra-arterial thrombolytics (eg., recombinant tissue plasminogen activator [rtPA] ) can be beneficial and alter the outcome significantly in patients presenting with hyperacute stroke. ${ }^{1-2}$ While nonenhanced CT can detect "early signs" of infarction and exclude hemorrhage, it cannot reliably demonstrate irreversibly damaged brain tissue in hyperacute ischemic stroke. ${ }^{3}$ Non-contrast CT is complemented by CT angiography (CTA) and CT perfusion (CTP) in some stroke protocols. Computed tomogram angiography (CTA) can detect stenosis or occlusion of extra- and intracranial arteries helping in management decisions. ${ }^{3}$ Computed tomogram perfusion (CTP) can provide information about brain perfusion, thus permitting differentiation of irreversibly damaged brain tissue from reversibly impaired "tissue at risk". ${ }^{4}$ Multiphasic CTP is also comparable to diffusion and perfusion MRI for predicting final infarct volume, infarct growth, and clinical severity in acute ischemic stroke. ${ }^{5}$ Multidetector CT thus allows the combined use of all three imaging modalities- non-enhanced CT, CTA, and CTP -to rapidly obtain comprehensive information regarding the extent of ischemic damage in acute stroke patients. ${ }^{3}$

From the Department of Diagnostic Imaging, The Ottawa Hospital, Ottawa, Canada ReCEIVED JANuARY 7, 2005. ACCEPTED IN FINAL FORM June 3, 2005. Reprint requests to: Mayank Goyal, Division of Neuroradiology, Department of Diagnostic Imaging, The Ottawa Hospital, Civic Campus, Ottawa, Ontario, Canada K1Y 4E9 
Magnetic resonance imaging (MRI) using diffusion weighted imaging (DWI) and perfusion weighted imaging (PWI) has been shown to be a reliable diagnostic tool in hyperacute ischemic stroke. ${ }^{6,7}$ However, some authors have raised doubt about the feasibility, utility and cost-effectiveness of MRI. ${ }^{8}$ This may be because most patients with acute stroke present to community hospitals where CT is typically more accessible than MRI. ${ }^{9}$ Other potential hurdles with MRI in an acute stroke setting are movement artefacts (especially if patient is uncooperative), MR incompatible monitoring devices and increased acquisition time.

In most Canadian centers, CT is more readily available than MRI for imaging of hyperacute stroke. The time required to acquire and interpret functional imaging studies in the setting of acute stoke is important. The aim of our study was to determine the mean time for the acquisition, processing and interpretation of CTP and CTA images in patients presenting with hyperacute stroke at our institution. We also compared the processing and interpretation times of CTA and CTP studies amongst three groups of radiologists with varying degree of expertise, i.e., radiology residents, neuroradiology fellows and consultant neuroradiologists with at least five years of experience.

\section{MATERIALS AND METHODS}

From January 2002 - December 2003, 38 patients with acute ischemic stroke were evaluated using a multi-modal CT imaging protocol. Among these, ten patients were randomly selected for review. Their CT scans were retrieved from the picture archival and communication system (PACS) server onto the GE Advantage Windows workstation. The CT stroke protocol at our institution consists of non-contrast $\mathrm{CT}$ to rule out hemorrhage followed by CTA and CTP studies. On CTA, the arterial system from the aortic arch to the circle of Willis is evaluated using 100$120 \mathrm{ml}$ Iohexol (Omnipaque 300, Amersham Health) injected at a rate of $3 \mathrm{ml} / \mathrm{sec}$ and a $20-22$ seconds delay. The CTP is performed covering a $2 \mathrm{~cm}$ thick area at the level of the basal ganglia chosen by the radiologist present onsite and involves administration of $40-50 \mathrm{ml}$ of additional contrast. All scans were acquired on a multidetector CT (GE Lightspeed Plus, Milwaukee, Wisconsin).

The additional time taken for acquiring CTA and CTP studies after the initial non-contrast CT scan was calculated in each patient from the times registered on the last images of each series - non-enhanced CT, CTA and CTP studies. This time included the actual scan acquisition time and the delay involved in setup of the protocol for the CTA and CTP studies.

The readers included two radiology residents (PGY3, PGY4 with two and three years of radiology training, respectively -Group I), two neuroradiology fellows (Year 1, Year 2 - Group II) and four consultant neuroradiologists with at least five years of experience (Group III). They were each presented with a short clinical history of the patients and the CTA and CTP studies for processing and interpretation. Prior to their entry into the study, both the radiology residents were trained to process and interpret CTA and CTP studies in acute stroke with ten cases each. Both the fellows had experience with CTA and CTP studies in at least 15 cases each.

Each reader was permitted to view the non-contrast images prior to processing CTA and CTP studies. All readers were required to process and interpret the images (for the presence or absence of any major vessel thrombus and penumbra), and save the images to the workstation. Major vessel thrombus was defined as thrombus present within the distal intracranial internal carotid artery (ICA), M1/M2 segments of the middle cerebral artery (MCA) or A1 segment of the anterior cerebral artery (ACA) on CTA. Penumbra was defined as an area that showed normal cerebral blood volume (compared to the contralateral hemisphere) but decreased cerebral blood flow and increased mean transit time on CTP. No quantitative map analysis was performed on CTP.

An independent observer timed each reader and the individual times of each reader were recorded separately for CTA and CTP studies. The statistical difference in mean times for processing and interpretation of the studies between the three groups of readers was analyzed using the unbalanced ANOVA test. The interobserver variation in CTA interpretation was calculated (using the Kappa test) with respect to identification of thrombus in three separate segments- the distal intracranial internal carotid artery, M1/M2 segment of the MCA and A1 segment of the ACA. Interobserver variability in CTP interpretation was also calculated (using the Kappa test) with regard to diagnosis of presence or absence of penumbra.

\section{RESULTS}

There were eight male and two female patients in the study with a mean age of $67.6 \pm 18.9$ years. The mean additional time for acquiring both CTA and CTP studies in the ten patients after performance of the initial non-contrast CT scan was $14.6 \pm 5.9$ minutes (Range -7 to $26 \mathrm{~min}$, Median $=13.5 \mathrm{~min}$ ). The time taken for CTA processing and interpretation for Group I, II and III was $2.3 \pm 1.3 \mathrm{~min}, 1.6 \pm 0.4 \mathrm{~min}$ and $1.5 \pm 0.7 \mathrm{~min}$ respectively. The time required for CTP processing and interpretation by the same groups was $5.2 \pm 1.7 \mathrm{~min}, 4.5 \pm 1.5$ min and $4.1+1.1$ min respectively.

There was a statistically significant difference of means between Groups I and III in the CTA and CTP processing and interpretation times $(\mathrm{p}=0.02, \mathrm{p}=0.01$ respectively) but no statistical difference in means between Groups I and II ( $\mathrm{p}=0.15$, $\mathrm{p}=0.22$ respectively) or Groups II and III ( $\mathrm{p}=0.31, \mathrm{p}=0.30$ respectively). These results are illustrated in the Table .

In CTA studies, there was $100 \%$ agreement in identification of thrombus in the distal ICA (Kappa =1), $95 \%$ agreement in the M1/M2 segments (Kappa $=0.95,95 \%$ confidence interval 0.91 to 0.98 ) and $100 \%$ agreement in the A1 segment (Kappa $=$ 1). In CTP studies, there was good interobserver correlation for identification of penumbra (Kappa $=0.86,95 \%$ confidence interval -0.83 to 0.90$)$.

\section{DISCUSSION}

Marler et al in 2000 showed that if the NINDS (National Institute of Neurological Disorders and Stroke) rt-PA stroke trial treatment protocol is followed, patients treated with rt-PA within 90 minutes of stroke onset have increased odds of improvement at 24 hours and favorable 3-month outcome compared to patients treated later than 90 minutes. ${ }^{10}$ Thus, time plays a major role in management decisions in hyperacute stroke. In acute stroke, both CT (CTA and CTP) and MRI (using diffusion weighted imagesDWI, perfusion weighted images-PWI and MR angiography- 
Table: Processing and interpretation times of CTA and CTP studies

\begin{tabular}{l|c|c|c}
\hline & $\begin{array}{c}\text { CTA } \\
(\mathbf{m i n})\end{array}$ & $\begin{array}{c}\text { CTP } \\
(\mathbf{m i n})\end{array}$ & $\begin{array}{c}\text { Combined times } \\
(\mathbf{m i n})\end{array}$ \\
\hline Group I & $2.3 \pm 1.3$ & $5.2 \pm 1.7$ & $7.42 \pm 2.4$ \\
\hline Group II & $1.6 \pm 0.4$ & $4.5 \pm 1.5$ & $6.1 \pm 1.6$ \\
\hline Group III & $1.5 \pm 0.7$ & $4.1 \pm 1.1$ & $5.6 \pm 1.3$ \\
\hline Group I vs Group III & $\mathrm{p}=0.02$ & $\mathrm{p}=0.01$ & $\mathrm{p}=0.02$ \\
\hline Group II vs Group III & $\mathrm{p}=0.31$ & $\mathrm{p}=0.30$ & $\mathrm{p}=0.34$ \\
\hline Group I vs Group II & $\mathrm{p}=0.15$ & $\mathrm{p}=0.22$ & $\mathrm{p}=0.17$ \\
\hline
\end{tabular}

Group I - Residents, Group II - Fellows, Group III - Consultant Neuroradiologists

MRA) can help assess the extent of dead/salvageable tissue and the presence and degree of any vascular narrowing. ${ }^{11}$ Some authors feel that practice and experience with MR imaging can significantly reduce the time and effort required to perform this technique and make the assessment of patients with hyperacute stroke rapid and comprehensive. ${ }^{12}$ However, the ready availability of CT scanners and decreased propensity for motion related artifacts may make CT an attractive alternative.

There are several reports in the literature of the time required to perform and interpret perfusion MR and CT studies in the setting of hyperacute stroke. It is important to have a time efficient study in hyperacute stroke since the smallest delay in thrombolytic administration can alter the outcome. ${ }^{13}$ In a review article, Lev et $\mathrm{al}^{14}$ commented that CTA/CTP is fast and convenient, adding only approximately ten minutes to the noncontrast CT scan (NCCT) examination. They also said that the accuracy of CTA and CTP in diagnosing ischemia and localizing thrombus to proximal or distal intracranial vessels far exceeds that of clinical examination (including National Institutes of Health stroke scale use), facilitating triage of appropriate candidates to intra-arterial thrombolysis. ${ }^{14}$ Tatlisumak ${ }^{15}$ further reiterated the time efficiency of $\mathrm{CT}$ in the scenario of hyperacute stroke where every moment is of critical importance and pointed out that a 'stroke CT package' would require approximately ten minutes compared to 20 minutes for a 'stroke MRI package'. Also, most scanners may not be immediately available because of an ongoing imaging examination. In such a scenario, the typical wait for a CT scan is approximately five minutes while the wait in MRI might be up to a half hour. ${ }^{15}$

While some authors advocate CT as the best method of rapidly assessing the brain in hyperacute stroke, ${ }^{13}$ there are reports of ultrafast MR imaging techniques requiring less than 15 minutes of imaging time. ${ }^{16}$ Due to varying availability of both modalities worldwide, it is likely that CT and MRI would coexist for decades and the imaging method in stroke chosen according to the local conditions and patient characteristics. ${ }^{15}$

In our study the mean time for acquisition of CTA and CTP studies in acute stroke patients was approximately 15 minutes.
This included the actual scan acquisition time and the delays involved in setting up of CTA and CTP protocols. So we feel that CT is comparable, if not better, than MRI in quickly obtaining key diagnostic information for reaching a therapeutic decision in acute stroke. Also the time taken for CTA and CTP processing and interpretation was under ten minutes for all three groups of readers with variable expertise in Neuroradiology - residents, fellows and consultant neuroradiologists. In our experience the CT examination overall adds about 20-30 minutes from the time the non-contrast CT is performed to the time where a therapeutic decision can be made.

There is also the issue of interobserver variability in interpretation of studies in hyperacute stroke. Coutts et $\mathrm{al}^{17}$ evaluated the reliability of assessing the percentage of DWIPWI mismatch (considered a marker of tissue at risk of infarction) amongst various readers in a setting of acute stroke. They concluded that quantifying mismatch visually is reproducible within observers but not reliable among observers, thus raising doubts about using mismatch for clinical decision making and clinical trial enrollment. ${ }^{17}$ Fiebach et al $^{18}$ showed that diffusion weighted MR imaging has higher interobserver homogeneity and substantially higher sensitivity and accuracy than non-enhanced CT in hyperacute stroke. However, a study examining the variability of perfusion parameters amongst three different CT technologists after post-processing CTP source data showed a high degree of correlation between parenchymal regions of interest on the various perfusion maps. ${ }^{19}$ There was good interobserver correlation in interpretation of CTA and CTP examinations in our study. The only variability in CTA interpretation was in the M1/M2 segment of the MCA which may be due to the fact that our sample size is small and because most of the thrombi were seen in the MCA. Therefore, we feel that addition of CTA and CTP to the non enhanced CT in hyperacute stroke will decrease interobserver variability and improve diagnostic accuracy.

In conclusion, we feel that CTA and CTP studies can be performed, processed and interpreted quickly in an acute stroke setting. These studies could potentially help in planning therapy, 
though, at present, there are no phase III randomized controlled trials that have evaluated these studies using clinical endpoints. Also it can be performed by people with varying degrees of expertise in neuroradiology with little difference in times of processing and interpretation.

\section{REFERENCES}

1. NINDS, Tissue plasminogen activator for acute ischemic stroke. The National Institute of Neurological Disorders and Stroke rtPA Stroke Study Group. N Engl J Med 1995; 333:1581-1587.

2. Furlan A, Higashida R, Wechsler L, et al. Intra-arterial prourokinase for acute ischemic stroke. The PROACT II study: a randomized controlled trial. Prolyse in Acute Cerebral Thromboembolism. JAMA 1999; 282:2003-2011.

3. Tomandl BF, Klotz E, Handschu R, et al. Comprehensive Imaging of Ischemic Stroke with Multisection CT. Radiographics 2003; 23:565-592.

4. Mayer TE, Hamann GF, Baranczyk J, et al. Dynamic CT perfusion imaging of acutestroke. Am J Neuroradiol 2000; 21:1441-1449.

5. Na DG, Ryoo JW, Lee KH et al. Multiphasic perfusion computed tomography in hyperacute ischemic stroke: comparison with diffusion and perfusion magnetic resonance imaging. J Comput Assist Tomogr 2003; 27:194-206.

6. Tong DC, Yenari MA, Albers GW, et al. Correlation of perfusionand diffusion-weighted MRI with NIHSS score in acute $(<6.5$ hour) ischemic stroke. Neurology 1998; 50:864-870.

7. Barber PA, Darby DG, Desmond PM et al. Prediction of stroke outcome with echoplanar perfusion- and diffusion-weighted MRI. Neurology 1998; 51:418-426.

8. Powers WJ, Zivin J. Magnetic resonance imaging in acute stroke: not ready for prime time. Neurology 1998; 50:842-843.

9. Schramm P, Schellinger PD, Klotz E, et al. Comparison of perfusion computed tomography and computed tomography angiography source images with perfusion-weighted imaging and diffusionweighted imaging in patients with acute stroke of less than 6 hours' duration. Stroke 2004; 35:1652-1658.
10. Marler JR, Tilley BC, Lu M, et al. Early stroke treatment associated with better outcome: the NINDS rt-PA stroke study. Neurology 2000; 55:1649-1655

11. Schaefer PW, Hunter GJ, He J, et al. Predicting cerebral ischemic infarct volume with diffusion and perfusion MR imaging. Am J Neuroradiol 2002; 23:1785-1794.

12. Schellinger PD, Jansen O, Fiebach JB, et al. Feasibility and practicality of MR imaging of stroke in the management of hyperacute cerebral ischemia. Am J Neuroradiol 2000; 21:11841189.

13. Pexman JH, Hill MD, Buchan AM, et al. Hyperacute stroke: experience essential when reading unenhanced CT scans. Am J Neuroradiol 2004; 25:516; author reply 516-518.

14. Lev MH, Nichols SJ. Computed tomographic angiography and computed tomographic perfusion imaging of hyperacute stroke. Top Magn Reson Imaging 2000; 11:273-287.

15. Tatlisumak T. Is CT or MRI the method of choice for imaging patients with acute stroke? Why should men divide if fate has united? Stroke 2002; 33:2144-2145.

16. Sunshine JL, Tarr RW, Lanzieri CF, et al. Hyperacute stroke: ultrafast MR imaging to triage patients prior to therapy. Radiology 1999; 212:325-332.

17. Coutts SB, Simon JE, Tomanek AI, et al. Reliability of assessing percentage of diffusion-perfusion mismatch. Stroke 2003; 34: 1681-1683.

18. Fiebach JB, Schellinger PD, Jansen O, et al. CT and diffusionweighted MR imaging in randomized order: diffusion-weighted imaging results in higher accuracy and lower interrater variability in the diagnosis of hyperacute ischemic stroke. Stroke 2002; 33:2206-2210.

19. Fiorella D, Heiserman J, Prenger E, Partovi S. Assessment of the reproducibility of postprocessing dynamic $\mathrm{CT}$ perfusion data. Am J Neuroradiol 2004; 25:97-107. 